\title{
Orthogonal Basis Expansion based Atrial Activity Reconstruction for Atrial Fibrillation Electrocardiogram Analysis
}

\author{
Sandun Kodituwakku, Thushara D. Abhayapala, Rodney A. Kennedy
}

\begin{abstract}
We propose a novel algorithm for extracting atrial activity from single lead electrocardiogram (ECG) signal sustained with atrial fibrillation (AF), based on a short-time expansion of an orthogonal basis function set. The method preserves the time variation of spectral content of the underlying AF signal, thus time-frequency analysis of the AF signal can be successfully performed. The new method is compared to the standard average beat subtraction (ABS) method using synthetic AF sustained ECG data. The orthogonal basis expansion method has a higher correlation with the original AF signal compared to the ABS method for a range of signal to noise ratio (SNR) levels, and correlation is improved by $16 \%$ at an SNR of OdB. Time-frequency analysis of the reconstructed AF signal based on Bessel distribution also shows the superiority of the orthogonal basis expansion method over ABS.
\end{abstract}

\section{INTRODUCTION}

AF is the most common sustained cardiac arrhythmia, increasing in prevalence with age, accounting for approximately one third of hospitalizations for cardiac rhythm disturbances [1]. AF is characterized by the replacement of consistent P-waves on the ECG by rapid oscillations (fibrillatory waves) that vary in amplitude, frequency, and shape, associated with an irregular ventricular response. AF affects approximately $10 \%$ of the population over age of 75 and is associated with an increased risk of stroke [1], [2].

ECG can be used as a noninvasive way of analyzing AF for characterizing paroxysmal and persistent AF conditions, and monitoring the response to cardioversion or drug induced treatment methods, with inherit advantages of being low cost, minimal risk, and ability to record for long period of times via Holter monitors [3], [4]. Analysis of AF and other supraventricular tachyarrhythmia conditions require cancelation of the ventricular activity (QRS complex and T-wave) from the ECG. Due to the overlapping spectral contents of atrial and ventricular activities, linear filtering is not possible, and also the higher dominance of ventricular activity in the ECG makes the extraction of atrial activity non-trivial [5], [6].

Two types of methods have been used in the literature for extracting atrial activity from the ECG. First type of methods, known as average beat subtraction (ABS) methods, work by estimating an average morphology of QRS complex and Twave, and subtracting the average template from individual

Sandun Kodituwakku sandun.kodituwakku@anu.edu.au, Thushara D. Abhayapala thushara. abhayapala@anu. edu . au, and Rodney A. Kennedy rodney. kennedy a anu. edu. au are with Applied Signal Processing Group, Research School of Information Sciences and Engineering, The Australian National University, Canberra, ACT 0200, Australia.
QRS-T wave with possible temporal and spacial alignment and scaling. ABS methods can be used with both single-lead and multi-lead ECG, but work on the assumptions that time invariance of wave morphology, and short-time stationarity of the signal. These assumptions often break in the presence of ectopic beats, and movement of electrodes, thus significant amount of residual QRS-T can be present in the extracted atrial signal [7]. The performance could be further limited for signals with short durations, as constructing a good QRS-T template become difficult [8].

Second type of methods, known as blind source separation methods, explore the spatial diversity of multi-lead ECG, and rely on the assumptions linear mixing of uncoupled atrial and ventricular activities. Techniques such as independent component analysis, and principal component analysis were employed with non-Gaussianity criteria is used for the separation of atrial and ventricular sources [6], [9]. Even though these methods estimate a global atrial activity using multiple leads, the performance significantly reduces for fewer number of leads. Especially for Holter recordings where only few electrodes are available, performance of the source separation techniques are not satisfactory [10].

Following the extraction of atrial activity, the spectral analysis or the time-frequency analysis is performed to identify the dominant AF frequency and its time variation [3], [5]. But both the above described methods do not take into account preserving the spectral content of the underlying AF signal during the process of atrial activity extraction. In this paper, we propose a novel method for reconstructing the atrial activity from AF sustained ECG by mode limited short-time expansion of an orthogonal basis function set. Atrial activity during the QRS-T duration was estimated by interpolating immediately preceding and following segments of AF signal. The method preserves the morphology and frequency content of the underlying AF signal, and is free from any QRS-T residues. The proposed algorithm is described in Section II, and performance evaluation of the new algorithm is given in Section III in terms of correlation at different SNR levels, and by construction of time-frequency plots. A comparison is also done between the proposed method and the ABS method.

\section{METHOD}

\section{A. Orthogonal Basis based AF Reconstruction}

Let $s(t)$ be a single lead AF sustained ECG signal. A typical segment of $s(t)$ is shown in Figure 1. Timings of starting point of QRS complex, and end point of T-wave of $i^{\text {th }}$ beat were named $Q_{i}$, and $P_{i+1}$ respectively. AF signal is clearly visible in the intervals $\left(P_{i}, Q_{i}\right)$, and $\left(P_{i+1}, Q_{i+1}\right)$, 
but is corrupted by the ventricular activity in the interval $\left(Q_{i}, P_{i+1}\right)$. In the following algorithm we use the known segments of the AF signal in $\left(P_{i}, Q_{i}\right)$ and $\left(P_{i+1}, Q_{i+1}\right)$ to estimate the ventricular corrupted segment in $\left(Q_{i}, P_{i+1}\right)$ by modeling the AF signal as a short-time weighted sum of orthogonal basis function set.

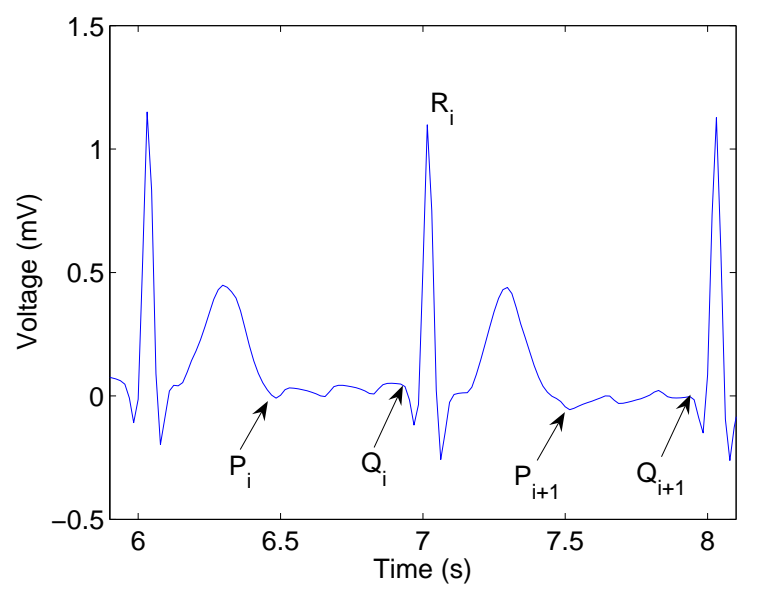

Fig. 1: A typical AF sustained ECG segment with nomenclature used in this paper.

Let $f_{i}(t)$ be the underlying AF signal over the interval $\left(P_{i}, Q_{i+1}\right)$. We represent $f_{i}(t)$ by a set of orthogonal basis functions $\left\{\phi_{n}(t)\right\}$. Due to the frequency modulated harmonic nature of the AF signal, the natural choice for the basis set was considered to be the complex exponential functions,

$$
\phi_{n}(t)=e^{j 2 \pi n t / T}
$$

where $T=Q_{i+1}-P_{i}$. Assuming that $f_{i}(t)$ is mode limited to $N$ with respect to $\left\{\phi_{n}(t)\right\}$,

$$
f_{i}(t)=\sum_{n=-N}^{N} a_{(n, i)} \phi_{n}(t) \quad P_{i} \leq t \leq Q_{i+1},
$$

where $\left\{a_{(n, i)}\right\}$ are the localized coefficients of basis functions. The limited number of modes which captures the underline AF signal will preserve the spectral content of the signal, and improve the noise robustness. By using $\left(P_{i}, Q_{i}\right)$ and $\left(P_{i+1}, Q_{i+1}\right)$ intervals of $s(t)$ to estimate $\left\{a_{(n, i)}\right\}$, we get

$\left(\begin{array}{c}s\left(P_{i}\right) \\ \vdots \\ s\left(Q_{i}\right) \\ s\left(P_{i+1}\right) \\ \vdots \\ s\left(Q_{i+1}\right)\end{array}\right)=\left(\begin{array}{ccc}\phi_{-N}\left(P_{i}\right) & \ldots & \phi_{N}\left(P_{i}\right) \\ \vdots & \ddots & \vdots \\ \phi_{-N}\left(Q_{i}\right) & \ldots & \phi_{N}\left(Q_{i}\right) \\ \phi_{-N}\left(P_{i+1}\right) & \ldots & \phi_{N}\left(P_{i+1}\right) \\ \vdots & \ddots & \vdots \\ \phi_{-N}\left(Q_{i+1}\right) & \ldots & \phi_{N}\left(Q_{i+1}\right)\end{array}\right)\left(\begin{array}{c}a_{(-N, i)} \\ \vdots \\ a_{(N, i)}\end{array}\right)$

which is written in short

$$
\mathbf{s}=\Phi \mathbf{a} .
$$

If $\left(Q_{i}-P_{i}\right)+\left(Q_{i+1}-P_{i+1}\right)>2 N+1$, then the system in (4) is over-determined, and usually this will be the case provided the ECG signal is sufficiently sampled. In other case, the system in (4) is under-determined. In either case, regularization can be used to overcome the problems associated with the over-determined or under-determined system. Regularization avoids modeling of the noise, thus effectively acts as a noise filter. It also guarantees a bounded solution, thus any remaining QRS-T residues will be suppressed. Tikhonov regularization [11] was applied in solving (4), and the solution can be written in the form

$$
\hat{\mathbf{a}}=\left(\Phi^{T} \Phi+\lambda^{2} \mathbf{I}\right)^{-1} \Phi^{T} \mathbf{s},
$$

where $\mathbf{I}$ is the identity matrix, $\lambda$ is the regularization parameter, and $T$ stands for the transpose of the matrix. Once a is estimated, the underlying AF signal during the $i^{t h}$ beat, $f_{i}(t)$ can be obtained by

$$
\hat{f}_{i}(t)= \begin{cases}s(t) & P_{i} \leq t \leq Q_{i} \\ \sum_{n=-N}^{N} \hat{a}_{(n, i)} \phi_{n}(t) & Q_{i}<t<P_{i+1} \\ s(t) & P_{i+1} \leq t \leq Q_{i+1} .\end{cases}
$$

Known AF segments are replaced by the original ECG, and ventricular corrupted segment is estimated by the basis function set. The whole AF signal $f(t)$ can be reconstructed by iterating the above process for each single beat.

\section{B. Time-Frequency Analysis}

Once the AF signal is estimated, time-frequency analysis was performed in order to identify the dominant AF frequency, and its time variation. Bessel kernel based timefrequency distribution [12] has shown a good frequency resolution, and error robustness in analyzing AF signals [13], and is given by

$$
\begin{aligned}
& B(t, \omega ; \phi)=\frac{1}{2 \pi} \iiint e^{j \xi \mu-j \tau \omega-j \xi t} \phi(\xi, \tau) \\
& \times f\left(\mu+\frac{\tau}{2}\right) f^{*}\left(\mu-\frac{\tau}{2}\right) d \xi d \mu d \tau,
\end{aligned}
$$

where $f(\mu)$ is the AF signal to be analyzed, $t$ and $\omega$ are time and frequency respectively, and $\tau$ and $\xi$ are time lag and frequency lag respectively. Limits of each integral is from $-\infty$ to $\infty$. The kernel function $\phi(\xi, \tau)$ for the Bessel distribution is given by

$$
\phi(\xi, \tau)=\frac{J_{1}(2 \pi \alpha \xi \tau)}{\pi \alpha \xi \tau},
$$

where $J_{1}(\cdot)$ is the Bessel function of first kind of order one, and $\alpha>0$ is a scaling factor.

\section{RESULTS}

\section{A. Synthetic AF sustained ECG}

The algorithm was applied to synthetic ECG signals generated from a dynamical model of motion [14] which was then modified by removing the P-waves, and adding simulated AF signal. Figure 2(a) shows the simulated ECG for normal sinus rhythm, and Figure 2(b) shows the AF sustained ECG. 
The AF signal was mathematically modeled by a sum of frequency modulated sinusoidals with time varying amplitude and its harmonics [7], [13] and is given by

$$
g(t)=\sum_{k=1}^{M+1} a_{k}(t) \cos \left[k \omega_{0} t+\frac{\Delta \omega}{\omega_{f}} \sin \left(\omega_{f} t\right)\right]+n(t),
$$

where $a_{k}(t)=e^{-\gamma(k-1)}\left(a+\Delta a \sin \left(\omega_{a} t\right)\right), \omega_{0}$ is the fundamental AF frequency, $\omega_{f}$ is the frequency of frequency modulation, $\Delta \omega$ is the maximum frequency deviation, $M$ is the number of harmonics excluding the fundamental, $\gamma$ is the decaying factor of harmonics, $a$ is the average amplitude of the fundamental, $\omega_{a}$ is the frequency of amplitude modulation, and $\Delta a$ is the maximum amplitude deviation. $n(t)$ represents white Gaussian noise, artifacts from insufficient QRS-T cancelation, and other ECG artifacts. According to the model, AF frequency is given by

$$
\omega_{A F}(t)=\omega_{0}+\Delta \omega \cos \left(\omega_{f} t\right) .
$$

A segment of simulated AF signal is shown in Figure 3(a) for $\omega_{0}=2 \pi 6 \mathrm{rad} / \mathrm{s}, \omega_{f}=2 \pi 0.04 \mathrm{rad} / \mathrm{s}, \Delta \omega=2 \pi 2 \mathrm{rad} / \mathrm{s}, M=3$, $\gamma=1, a=0.02 \mathrm{mV}, \omega_{a}=2 \pi 0.08 \mathrm{rad} / \mathrm{s}, \Delta a=0.005 \mathrm{mV}$.

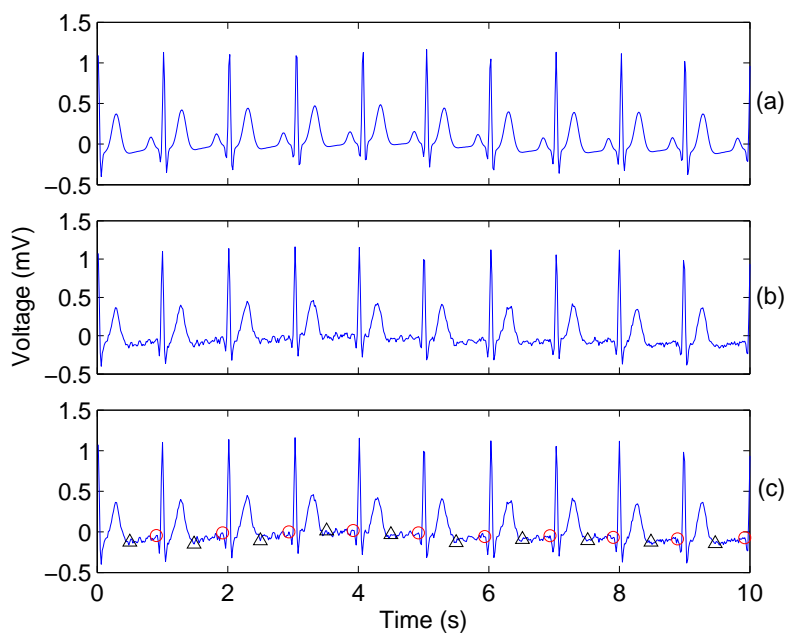

Fig. 2: (a) Synthetic ECG for normal sinus rhythm [14], (b) P-wave was removed from normal sinus rhythm and AF signal (9) was superimposed, (c) Fiducial points $P_{i}$ and $Q_{i}$ were accurately identified.

\section{B. Data Processing}

Once AF sustained ECG was generated, baseline wander was removed by passing through a high-pass filter with $0.5 \mathrm{~Hz}$ cut off frequency. Then, R-peaks were detected using Hilbert transform based peak detection technique [15]. Following R-peak detection, fiducial points $P_{i}$ and $Q_{i}$ were identified by $P_{i}=R_{i-1}+0.5 \overline{R R}$, and $Q_{i}=R_{i}-0.1 \overline{R R}$, where $\overline{R R}$ is the mean $R R$ interval. Accurate identification of fiducial points $P_{i}$ and $Q_{i}$ is shown in Figure 2(c).

Following identification of fiducial points, orthogonal basis expansion based algorithm described in Section II was applied to reconstruct the AF signal from the ECG. Number of modes $N=16$, and regularization parameter $\lambda=1.8$ were used to maximize the correlation with the original $\mathrm{AF}$ signal. ABS method was also applied to the same ECG data in order to compare the performance of the proposed method. ABS method involved constructing a mean QRS-T template, and subtracting the QRS-T template from individual beat after temporal alignment and scaling with each R-peak. Figure 3 shows the simulated AF from the model (9), and reconstructed AF signals using orthogonal basis expansion method, and ABS method. QRS-T residues are apparent in ABS method at times $t=6,7,8 s$, where as orthogonal basis expansion method has successfully suppressed all the ventricular activity.

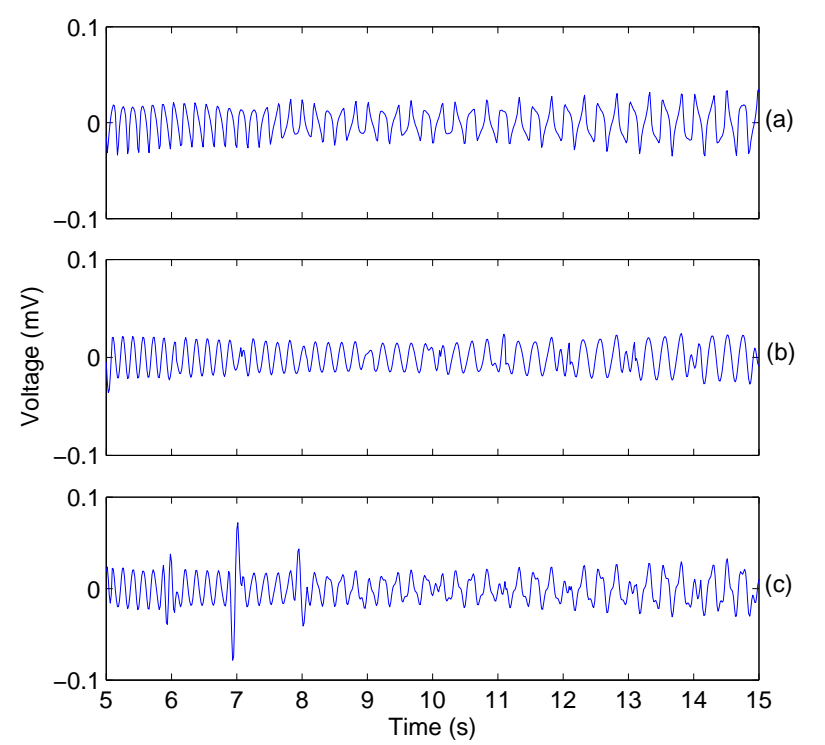

Fig. 3: (a) Simulated AF signal (9), (b) AF signal reconstructed using orthogonal basis expansion, (c) AF signal reconstructed using average beat subtraction.

\section{Performance Evaluation}

Correlation coefficient was used to evaluate the performance of the proposed method, and is given by

$$
C C=\frac{\left[\int_{-\infty}^{\infty} f(\tau) g^{*}(\tau-t) d \tau\right]^{2}}{\left[\int_{-\infty}^{\infty} f(\tau) f^{*}(\tau-t) d \tau\right]\left[\int_{-\infty}^{\infty} g(\tau) g^{*}(\tau-t) d \tau\right]},
$$

where $g(t)$ is the simulated AF signal, and $f(t)$ is the reconstructed AF signal using a particular method. Figure 4 shows the correlation coefficients for orthogonal basis expansion method and ABS method, under white Gaussian noise, for a range of SNR settings. It is clear that proposed method outperforms ABS for all the noise settings, and at OdB SNR correlation was improved by $16 \%$.

Then, time-frequency analysis was performed on reconstructed AF signals using Bessel kernel based time-frequency distribution. Figure 5 shows the time-frequency plots for simulated AF (9), and reconstructed AF signals from orthogonal basis expansion, and ABS methods. It is evident 


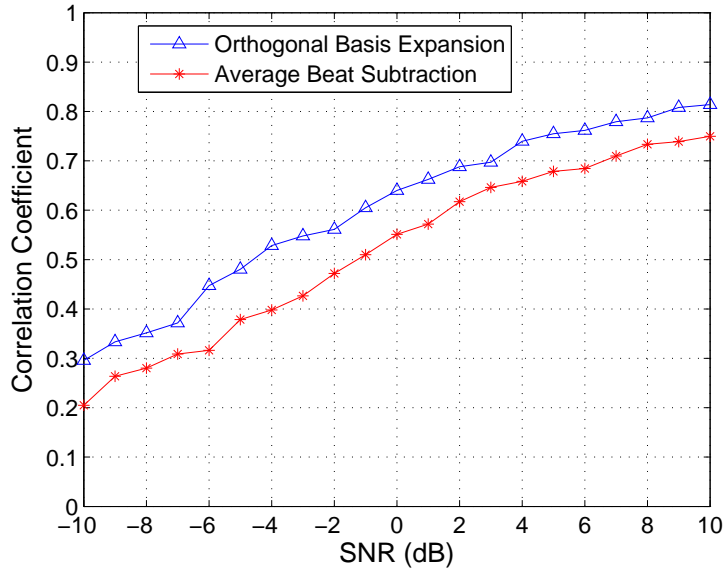

Fig. 4: Correlation coefficients of reconstructed AF signal using orthogonal basis expansion and average beat subtraction with respect to the simulated AF signal (9) under additive white Gaussian noise.

that the ABS method is vulnerable to QRS-T residues, and time-frequency plot gives discontinuities, and misleading frequencies at points where QRS-T residues are remaining. On the other hand, proposed orthogonal basis expansion method has a smoother frequency trend, and more closely matches with the original AF signal.

\section{CONCLUSIONS}

A novel algorithm based on mode limited short-time expansion of an orthogonal basis function set was proposed for extracting the atrial activity from the ECG under AF condition. Correlation coefficients indicate that the proposed method outperforms the standard ABS method for a range of SNR levels. Regularization and limited number of modes make the proposed method less vulnerable to noise, and preserves the spectral content of the underlying AF signal, thus results in a better time-frequency representation. Finding the values for optimum number of modes, and optimum regularization parameter remains an open question, and training and cross validation based methods need to be applied in order to find optimal values of these parameters.

\section{REFERENCES}

[1] V. Fuster, L. E. Ryden, and et al., "Acc/aha/esc 2006 guidelines for the management of patients with atrial fibrillation," Amer. J. Cardiol., vol. 48(4), pp. 854-906, Aug 2006.

[2] S. N. Singh, X. C. Tang, and et al., "Quality of life and exercise performance in patients in sinus rhythm versus persistent atrial fibrillation," Amer. J. Cardiol., vol. 48(4), pp. 721-730, Aug 2006.

[3] A. Bollmann, N. A. Kanuru, K. K. McTeague, P. F. Walter, D. B. DeLurgio, and J. J. Langberg, "Frequency analysis of human atrial fibrillation using the surface electrocardiogram and its response to ibutilide," Amer. J. Cardiol., vol. 81, pp. 1439-1445, Jan 1998.

[4] S. Petrutiu, J. Ng, G. M. Nijm, H. Al-Angari, S. Swiryn, and A. V. Sahakian, "Atrial fibrillation and waveform characterization: A time domain perspective in the surface ECG," IEEE Eng. in Med. and Bio. Mag., vol. 25, no. 6, pp. 24-30, 2006.
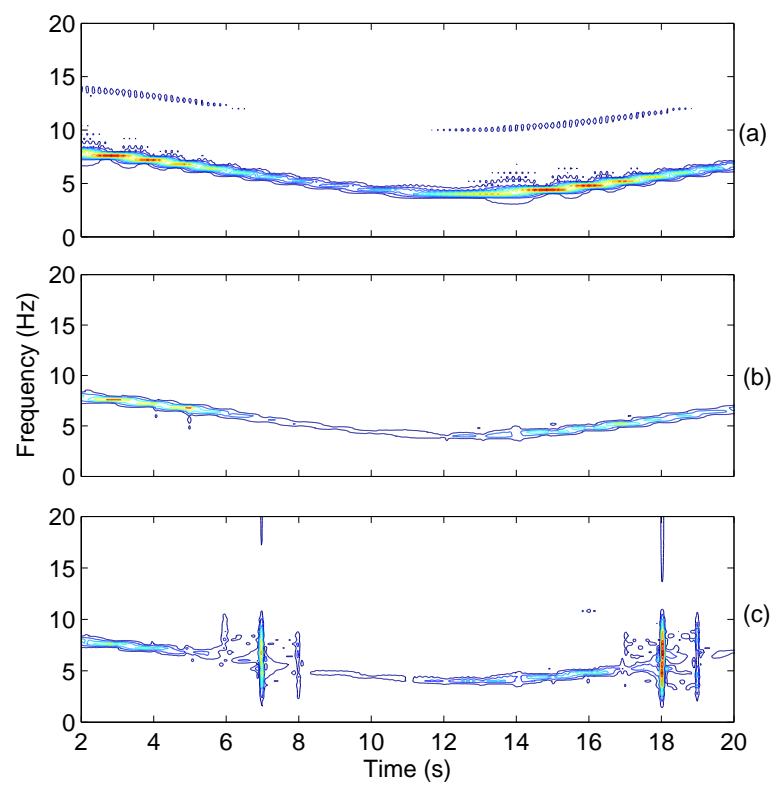

Fig. 5: Bessel kernel based time-frequency distributions for (a) simulated AF signal (9), (b) reconstructed AF signal using orthogonal basis expansion, (c) reconstructed AF signal using average beat subtraction.

[5] A. Bollmann, D. Husser, L. Mainardi, F. Lombardi, P. Langley, A. Murray, J. J. Rieta, J. Millet, S. B. Olsson, M. Stridh, and L. Sornmo, "Analysis of surface electrocardiograms in atrial fibrillation: techniques, research, and clinical applications," Europace, vol. 8, no. 11, pp. 911-926, 2006.

[6] J. Rieta, F. Castells, C. Sanchez, V. Zarzoso, and J. Millet, "Atrial activity extraction for atrial fibrillation analysis using blind source separation," IEEE Trans. Biomed. Eng., vol. 51, no. 7, pp. 1176-1186, 2004 .

[7] M. Stridh and L. Sornmo, "Spatiotemporal QRST cancellation techniques for analysis of atrial fibrillation," IEEE Trans. Biomed. Eng., vol. 48(1), pp. 105-111, Jan 2001.

[8] M. Lemay, J. M. Vesin, A. van Oosterom, V. Jacquemet, L. Kappenberger, and E. P. F. de Lausanne, "Cancellation of ventricular activity in the ECG: Evaluation of novel and existing methods," IEEE Trans. Biomed. Eng., vol. 54, no. 3, pp. 542-546, 2007.

[9] F. Castells, J. Rieta, J. Millet, and V. Zarzoso, "Spatiotemporal blind source separation approach to atrial activity estimation in atrial tachyarrhythmias," IEEE Trans. Biomed. Eng., vol. 52, no. 2, pp. 258267, 2005.

[10] R. Alcaraz and J. J. Rieta, "Adaptive Singular Value QRST Cancellation for the Analysis of Short Single Lead Atrial Fibrillation Electrocardiograms," Proc. Computers in Cardiology, vol. 34, pp. 513516, 2007.

[11] A. Ribes and F. Schmitt, "Linear inverse problems in imaging," IEEE Sig. Proc. Mag., vol. 25, no. 4, pp. 84-99, 2008.

[12] Z. Guo, L. Durand, and H. C. Lee, "The time-frequency distributions of nonstationary signals based on a bessel kernel," IEEE Trans. Sig. Proc., vol. 42, no. 7, pp. 1700-1707, Jul 1994.

[13] S. Kodituwakku, T. D. Abhayapala, and R. A. Kennedy, "Atrial fibrillation analysis using bessel kernel based time frequency distribution technique," Proc. Computers in Cardiology, vol. 35, pp. 837-840, Sep 2008.

[14] P. McSharry, G. Clifford, L. Tarassenko, and L. Smith, "A dynamical model for generating synthetic electrocardiogram signals," IEEE Trans. Biomed. Eng., vol. 50, no. 3, pp. 289-294, 2003.

[15] D. S. Benitez, P. A. Gaydecki, A. Zaidi, and A. P. Fitzpatrick, "A new QRS detection algorithm based on the Hilbert transform," Proc. Computers in Cardiology, vol. 27, pp. 379-382, 2000. 